\title{
Two-Dimensional Seven-State Potts Model Under External Magnetic Field
}

\author{
T. ÇELİK, Y. GÜNDÜÇ and M. AYDIN \\ Hacettepe University, Physics Department \\ 06532 Beytepe, Ankara, Turkey
}

\begin{abstract}
The two-dimensional Potts Model with seven states under external field is studied using a cluster algorithm. Cluster size distribution and the fluctuations in the average cluster size provide helpful information on the order of phase transitions.
\end{abstract}

Potts model [1] is known to have a very rich critical behaviour and considered as a testing ground for both analytical and numerical methods. Recently, the problem is mainly focused on determining the nature of the phase transition, especially the weak first-order transition occuring in this model [2, 3]. In two dimensions, the Potts model displays a second-order phase transition for the number of states $q=2,3$ and 4 , and first-order transition for $q \geq 5$, where transitions become stronger as $q$ increases [ [f, 5]. One of the most reliable methods to study first-order transitions is to observe the doubly-peaked probability distribution for energy [6, 7]. At the critical point, the order parameter and the cluster size distribution should also exhibit the same behaviour. A first-order phase transition however is expected to become weaker under an increasing external field and it becomes a difficult task to recognize a double-peak in a weak first-order phase transition. Further increase in external field reduce the strength of the transition and the phase transition disappears for large external field strengths. This process is the result of two competing interactions: spins form sizable clusters in some regions because of the tendency to align in the field direction, while the thermal fluctuations result in disintegration of the clusters. The external field yields the ordering of the system at higher temperatures, shifting the critical temperature to higher values as well as reducing fluctuations in the system. On the other hand, the dynamical changes associated with a phase transition in the system are reflected in the fluctuations and variations in the cluster size. The aim in this work is to observe, by using a cluster algorithm, the dynamical behaviour of cluster size variations with respect to temperature and the external field.

In the present work, the critical behaviour of the two-dimensional Potts model with seven states under external magnetic field is studied using cluster algorithm. Equilibrium averages, fluctuations in the average cluster size and the histograms for 
energy, order parameter and the average cluster size are obtained as a function of the temperature for different field strengths.

The Hamiltonian of the two-dimensional Potts model is given by

$$
\mathcal{H}=K \sum_{<i, j>} \delta_{\sigma_{i}, \sigma_{j}}+H \sum_{i} \delta_{\sigma_{i}, o} .
$$

Here $K=J / k T$; where $k$ and $T$ are the Boltzmann constant and the temperature respectively, and $J$ is the magnetic interaction between spins $\sigma_{i}$ and $\sigma_{j}$, which can take values $0,1,2, \ldots, q-1$ for the $q$-state Potts model and $H=h / k T$ with $h$ is the external field along the orientation 0 . Reader can refer to the review article by $\mathrm{Wu}$ [5] for detailed information about the model. Order of the transitions can be studied by calculating specific heat

$$
C=\frac{1}{k T^{2}}\left(<E^{2}>-<E>^{2}\right)
$$

and the Binder cumulant 8

$$
B=1-\frac{<E^{4}>}{3<E^{2}>}
$$

on finite lattices, where $E$ is the energy of the system.

The dynamical evolution of clusters and fluctuations in the observables are strongly dependent on the correlations in the system. When the temperature is high the clusters are small, and they start growing as the critical point is approached. If the correlation length in the system is finite (especially when it is shorter than the lattice size), existing large clusters may break down to smaller ones with thermal fluctuations in the system. Hence in such a system, very large and very small clusters may coexist, resulting in large fluctuations in the cluster size. If the correlation length is very large as in the case of weak first-order phase transition or infinite as in the case of a second-order transition, the existing large clusters can not easily disintegrate with thermal fluctuations. A similar effect can be seen when an external field is turned on in a system with first-order phase transition. The effect of the external magnetic field aligns the spins parallel to the field direction creating large clusters and preserve these clusters from the effects of thermal fluctuations. These considerations led us to the usage of the average cluster size, fluctuations in the cluster size, cluster size distribution as the operators to investigate the changes in the phase transition with respect to variations of temperature and external field strength. The cluster algorithm which introduces a global update by means of selecting clusters and updating all spins in the cluster proved to be very successful in eliminating critical slowing down and super critical slowing down as in the firstorder transitions in systems such as Potts [9] and $\mathrm{O}(\mathrm{N})$ [10] models. Studying any operator which uses information related to the clusters is extremely straightforward 
since this information is inherited in the cluster algorithm. The algorithm used in this work is similar to Wolf's algorithm, with the exception that, $H$ is incorporated following Dotsenko et al [11] and before calculating the observables, searching the clusters is continued until the total number of sites in all searched clusters is equal to or exceeds the total number of sites in the lattice.

In this work, the two-dimensional, $q=7$ Potts model with external magnetic

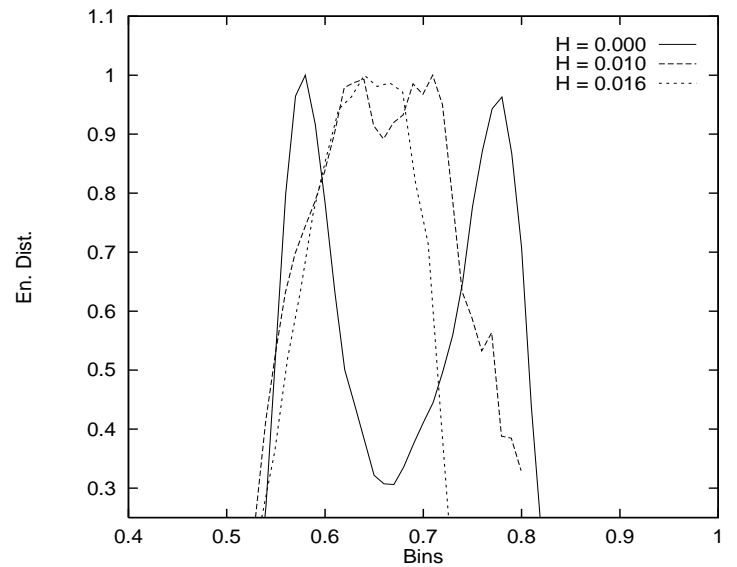

Fig. 1 . Energy histograms at the critical point $K_{c}$ for several values of $H$.

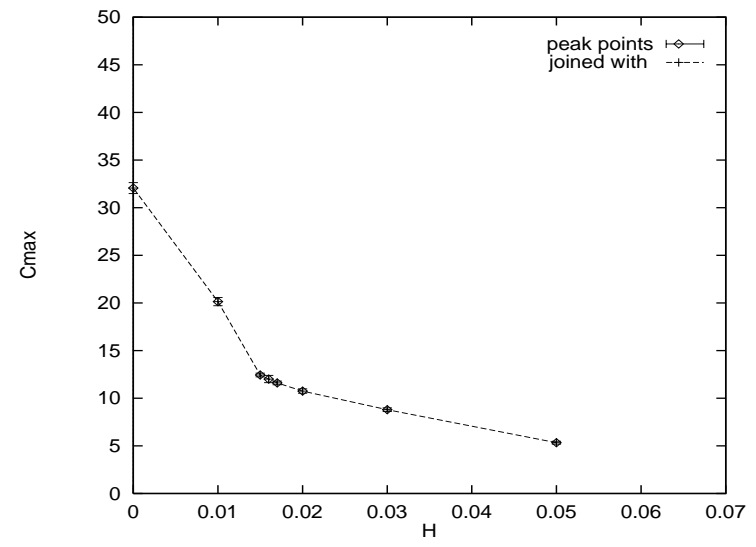

Fig. 2 . Specific heat peaks vs. $H$. Dashline is drawn to guide the eye.

field has been simulated on $64 \times 64$ square lattice. After thermalization with $10^{5}-2 \times 10^{5}$ sweeps, $5 \times 10^{5}-2 \times 10^{6}$ iterations are performed at different values of the coupling $K$ and the field $H$. Longer runs with up to $3 \times 10^{6}$ iterations are done near the finite-size critical value $K_{c}$ at each field strength $H$. For $H=0.00,0.01,0.015,0.016,0.02,0.03$ and 0.05 the long runs are performed at $K_{c}=1.2909,1.2774,1.2707,1.2696,1.2652,1.2531$ and 1.2299 respectively, where these points are chosen as the estimated peak positions of the specific heat. From these long runs we have evaluated by appropriate reweighting [12] the energy as a function of the temperature for each value of $H$. The continuous curves of energy, specific heat and the Binder cumulant obtained through extrapolation from one long run are in very good agreement with the data points over rather wide ranges of $K$ for each $H$ value. The point should be stressed here is that the success of an extrapolation depends on number of statistically independent configurations used, which is provided by the cluster algorithm employed here. As a first criterion to distinguish phase transition, we have checked for each value of $H$, the energy distribution at the critical temperature where the specific heat possess a peak. At $H=0$, where seven-state Potts model exhibits first-order character, a distinct double-peak is observed. As one can see from figure 1 , with increasing $H$ the double peak converges to a single peak while $H$ reaches to the value of 0.016 . Our simulations indicate that 
$H=0.016$ is almost at the verge of the first-order phase transition region and for larger values of $H$ we have seen no double peak behaviour but from the observation of a single gaussian energy distribution, it is hard to conclude whether the transition becomes second-order or it is totaly wiped out.

From specific heat peaks and the Binder cumulant minima, relevant information

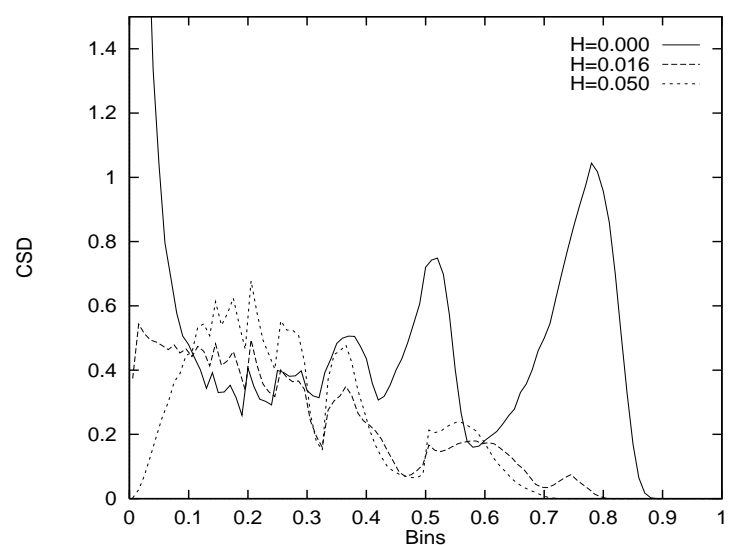

Fig. 3 . Cluster size distributions for several values of $H$.

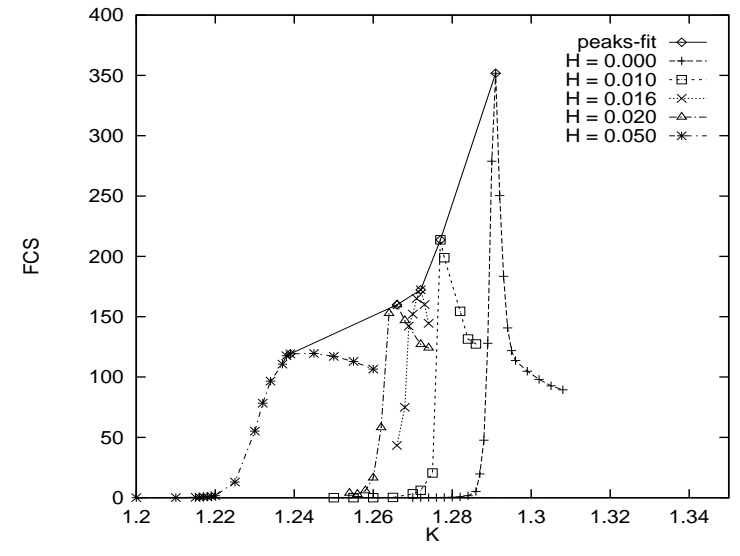

Fig. 4. Fluctuations in cluster size vs. $K$.

for the phase transition may also be obtained. For a first-order phase transition, the specific heat exhibits Dirac-delta function like shape. While the system moves towards the region of softer phase transitions with increasing $H$, the peak widens as well as the height is reduced. In the case of no phase transition, the system may still be expected to possess some fluctuations in the energy. For the set of values of the increasing external field, one obtaines a series of specific heat curves which become lower and wider as one moves towards the lower valus of $K$. Instead of a polynomial fit, here we have simply joined the tips of the successive specific heat curves by straight lines and displayed it in figure 2. This most naive presentation already prevails the existence of two distinct regimes and the turnover point is at $H=0.016$ where the line of first order transitions comes to an end. Our data on the Binder cumulant minima led to the same conclusion.

In order to have more microscopic insight and demonstrate what possibly the cluster search may add to the knowledge, we studied the time evolution of average cluster size (CS), its fluctuations (FSC) and the cluster size distributions (CSD). While the average energy fluctuates within a limited range about a mean value, the size of then existing clusters may easily fluctuate between the two extremes, namely from a single spin to a cluster of lattice size. Hence it seems to us that one may draw more information from cluster formation about the dynamical changes occuring in 
the system. Although the clusters we consider are of the Swendsen-Wang type, since the changes in cluster size are due to the update algorithm used, the observables not dealing with the exact cluster size (like $F C S$ and $C S D$ ) should give correct information on the system. We have studied the time evolutions over $2-3$ million iterations of average cluster sizes about $K_{c}$ for each value of the external field. These data are very much the same of the corresponding time-series for energy but more amplified and displays more insight about the grouping of spins leading to the concerned energy value. Two-state structures of first-order transitions and the long range fluctuations of second order phase transitions are more easily detectable comparing to the energy-time series. In figure 3, we show the corresponding cluster size distributions in terms of histograms over the cluster size (normalized to the volume) in the range of 0 to 1 . From figure 3 , one can easily follow the appearence of the two-state of small and large size clusters as for the $H=0.00$ case, almost equal weight for all sizes as for $H=0.016$ and loosing the small and large clusters in favor of medium sizes for $H>0.016$. The point to bring into the readers attention here is that the distribution of small clusters (bins $<0.1$ ) are very different for the three cases considered. For a first-order phase transition a substantial amount of small clusters are present and they gradually disappear while the phase transition weakens.

The fluctuations FCS $\left(F C S=<(C S)^{2}>-<C S>^{2}\right)$ in the average cluster size are calculated for different values of $H$, as a function of $K$. For the case of no external field, at small $K$ values (high temperatures) the cluster sizes are small, hence $F C S$ is small. As $K$ increases, the cluster sizes are getting larger, but $F C S$ increases due to the existence of the small-size clusters as well as the large ones. At the critical point, formation of the largest clusters leads to the largest value of $F C S$. One can make a similar discussion for $K>K_{c}$ (when the critical point is approached from above). When the external field is turned on, spin alignments in the field direction reduces the fluctuations and the probability of finding large and small clusters in coexistence decreases with the increasing magnetic field. FCS for $H=0.00,0.01,0.016,0.02$ and 0.05 are plotted in figure 4. Joining the tips of the successive FCS curves in figure 4 yields nothing but exactly what is displayed in figure 2 apart from a small shift in $K$ due to the finite size effects. As can be seen from these plots, increasing magnetic field reduces the critical temperature, as well as the peak heights. As $H$ increases, the $F C S$ curves widen and after some $H$ value, it is hard to see a distinct maximum point. At $H=0.05$ the $F C S$ curve is step function like where the phase transition is already wiped out. Besides the appearence of two different regimes on either side of the field value $H=0.016$, figure 4 also displays differences in the distinct shapes of the FCS curves belonging to two regimes. We observed that the $F C S$ curves for $H>0.016$ are continuous and a polynomial fit is possible which may be denoted as the sign of a smooth change in the average cluster size in the system. The curves for $H<0.016$ have the shape of a spike, for which the only possible polynomial fit is to have two distinct polynomials joining at 
the tip. This appearent discontinuity in the rate of fluctuations in cluster size may be attributed to the two state structure of first-order phase transitions. The low temperature asymptotic values for all $H$ seem to be almost the same regardless of the order of transition. Because of small thermal fluctuations at low temperatures, the clusters freeze after they are formed, resulting in a small FCS value.

In conclusion, one can see from $C S D$ and $F C S$ plots and from the energy histograms obtained near $K_{c}$ for each considered value of $H$ and from the extrapolations performed using the fitted values of the maximums of the specific heat, the first-order transition in 2D $q=7$ Potts model seems to disappear at $H=0.016$. What we would like to bring into attention here is that the observation of the temperature dependence of fluctuations in the average cluster size and the cluster size distributions gives valuable information about the nature of the transition and this kind of investigation may enrich the physical insight when employed in studying phase transitions.

Further work is planned to study random-field and random-bond Potts models using the same algorithm.

The support from TÜBİTAK through project TBAG-1299 is acknowledged. 


\section{References}

[1] POTTS R. B., Proc. Camb. Phil. Soc., 48 (1952) 106.

[2] BILlOIRE A., Nuc. Phys. (Proc. Suppl.), B42 (1995) 21.

[3] LEE J. and KOSTERLITZ J. M., Phys. Rev. Lett., 65 (1990) 137.

[4] BAXTER R. J., J. Phys., C6 (1973) L445.

[5] WU F. Y., Rev. Mod. Phys., 54 (1982) 235.

[6] BINDER K. and LANDAU D. P., Phys. Rev., B30 (1984) 1477.

[7] CHAlla M. S. S., LANDAU D. P. and BINDER K., Phys. Rev., B34 (1986) 1841.

[8] BINDER K., Phys. Rev. Lett., 47 (1981) 693.

[9] SWENDSEN R. H. and WANG J. S., Phys. Rev. Lett., 58 (1987) 86.

[10] WOLF U., Phys. Rev. Lett., 62 (1989) 361.

[11] DOtsenkO V. S., SELKE W. and TAlaPOV A. L., Physica, A170 (1991) 278.

[12] FerRenBerG A.M. and SWEndSEn R. H., Phys. Rev. Lett., 61 (1988) 2635. 


\section{FIGURE CAPTIONS}

Figure 1. Energy histograms at the critical point $K_{c}$ for several values of $H$.

Figure 2. Specific heat peaks vs. $H$. Dashline is drawn to guide the eye.

Figure 3. Cluster size distributions for several values of $H$.

Figure 4. Fluctuations in cluster size vs. $K$. 